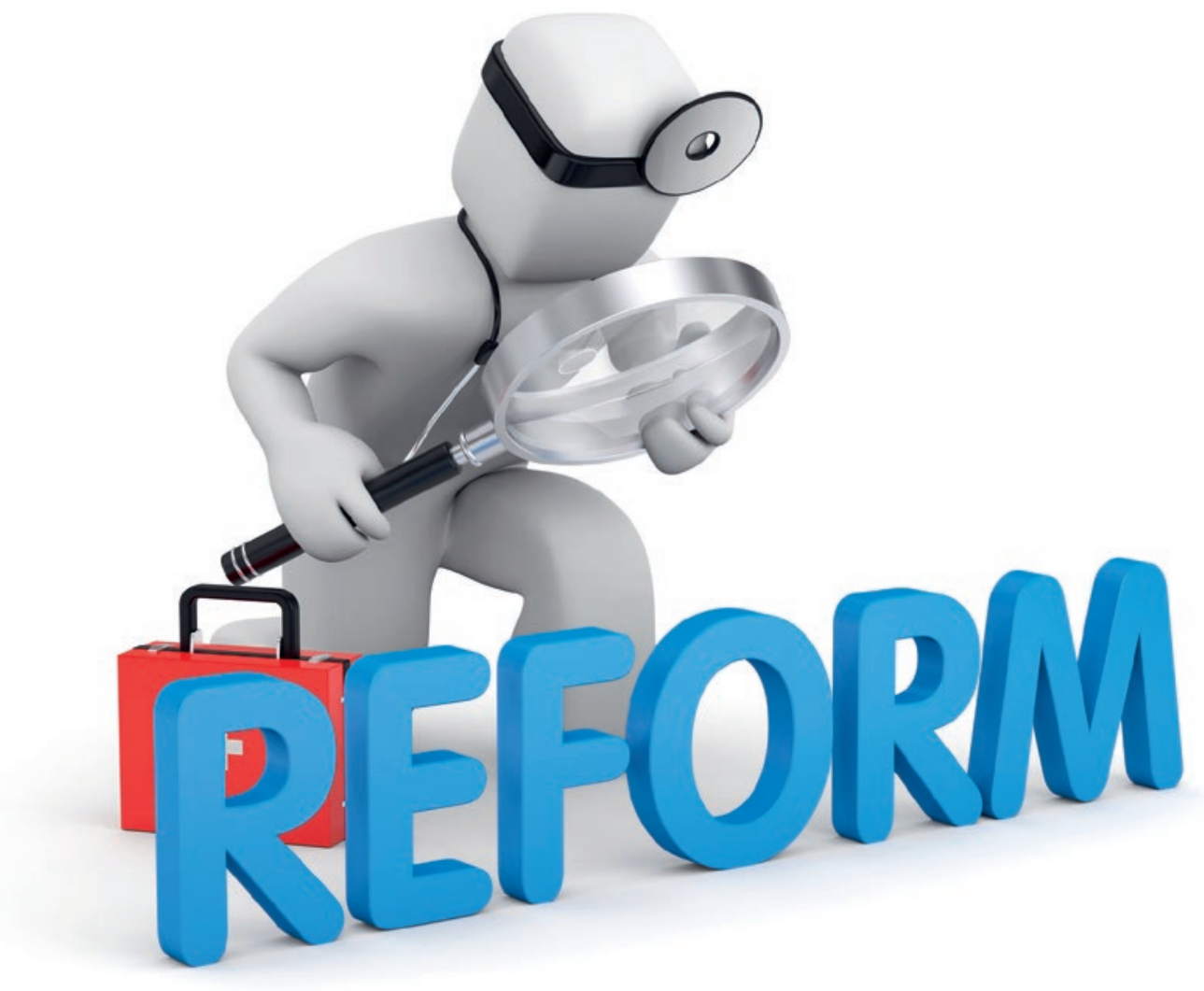

\title{
Zum Wohl der Patientinnen und Patienten
}

\section{Hans Stalder ${ }^{a}$, Beat Bürgenmeier}

a Prof. em. der Medizin, Universität Genf; b Prof. em. der Wirtschaftswissenschaften, Universität Genf

Finanzielle Fragen im Mittelpunkt, unterschiedliche Interessen der Beteiligten: Bisherige Vorschläge zur Reform des Gesundheitswesens überzeugen nicht. Wie könnte eine Umwandlung aussehen, die die Bedürfnisse der Patientinnen und Patienten ins Zentrum stellt und gleichzeitig eine Senkung der Kosten ermöglicht? Dieser Artikel* skizziert eine Lösung.

Das Gesundheitssystem in der Schweiz gilt allgemein als ausgezeichnet. Allerdings haben sich die Gesundheitskosten seit 1995 mehr als verdoppelt und sind im Verhältnis zum BIP von 9,3\% auf 11,9\% gestiegen. Die Prämien sind für Personen oder Familien mit niedrigem Einkommen immer schwerer zu ertragen und müssen teilweise oder sogar ganz vom Staat finanziert werden. Andere verzichten auf die Pflege aus finanziellen Gründen. Schliesslich haben Abrechnungs- und Qualitätskontrollsysteme zu einer Bürokratisierung geführt, die von allen Leistungserbringern im Gesundheitswesen als aufreibend empfunden wird. Zurzeit liegen verschiedene Vorschläge von Bundesrat und Mitgliedern des Parlaments zur Senkung der Kosten des Gesundheitssystems vor. Zwei Initiativen zielen ebenfalls darauf ab, sie zu reduzieren. Die Frage, wo gespart werden soll, hat einen erbitterten Kampf zwischen den verschiedenen beteiligten Akteuren ausgelöst.

Infolgedessen sind die Reformen im Gesundheitssystem kaum vorangekommen, weil die Interessen der verschiedenen Akteure gegensätzlich sind und die meisten Vorschläge nur den wirtschaftlichen Aspekt berücksichtigen. Unsere Vorschläge versuchen, der 
Reformdebatte durch eine andere Perspektive neue Impulse zu geben. Sie geht nicht von einer Analyse der Interessen der Akteure im Gesundheitssystem aus, sondern stellt die Patientinnen und Patienten in den Mittelpunkt der medizinischen Versorgung. Sie basiert auf einer integrierenden Sichtweise, neuen Formen der Finanzierung und auf einer unabhängigen Qualitätskontrolle. Durch Abbau der Bürokratie trägt sie zur Kostensenkung bei und verbessert die Arbeitsbedingungen. Schliesslich wird im vorliegenden Beitrag auf die wichtigsten Bedingungen hingewiesen, die für die Umsetzung der Reform erfüllt werden müssten.

\section{Ein personenzentriertes Gesundheits- system}

Die Idee, das Gesundheitssystem auf der Grundlage der Bedürfnisse der Patientinnen und Patienten zu reformieren, ist nicht neu. Es geht nicht darum, die Versorgung entsprechend den Interessen des einen oder anderen Akteurs zu reformieren, sondern sie patientenzentriert [1] oder noch besser, personenzentriert (Person centered care, PCC) [2] zu gestalten. In den Vereinigten Staaten sind Organisationen wie das «Patient Centered Medical Home» [3] oder die "Accountable Care Organizations» [4] am ehesten mit der PCC vereinbar. Wie kann man sich eine solche Organisation oder ein solches Netzwerk vorstellen, das durch das Bild eines «Medical Home» symbolisiert werden kann? Das «Medical Home» ist ein Konzept, das eine ganzheitliche Betreuung beinhaltet. Es kann «virtuell» mittels elektronisch verbundenen Netzwerken organisiert oder an einem einzigen Ort gruppiert sein. Die Person, die sich krank fühlt, sucht dort ihren Hausarzt auf. Im einfachsten Fall stellt der Arzt die Diagnose und verschreibt die Behandlung, und der oder die Betroffene geht wieder nach Hause. Aber meistens sind die Probleme komplizierter, insbesondere bei chronischen Erkrankungen oder Polymorbidität. Das «Medical Home» ermöglicht es, ergänzende Tests wie Labor und Röntgen durchzuführen, mithilfe von Pflegepersonal die Nachsorge zu organisieren, Anweisungen zur Vorbeugung und Behandlung zu geben, die Betroffenen an eine Spezialistin oder einen Spezialisten zu überweisen oder ins Krankenhaus zu schicken und gegebenenfalls Hauspflege oder soziale Unterstützung zu organisieren. Es ist die hausärztliche Equipe, die die Patientin oder den Patienten während der gesamten Krankheit begleitet. Die Hausärztin resp. der Hausarzt ist für das Funktionieren des «Medical Home» von entscheidender Bedeutung. Sie müssen nicht nur wissenschaftlich auf dem Laufenden und professionell sein, sondern auch die Fähigkeit haben, zu kommunizieren, zusammenzuarbeiten, zu lenken, zu koordinieren und die Gesundheit zu fördern. Das «Medical Home» bietet daher alle Aspekte der Patientenversorgung und verbessert das ärztliche Vertrauensverhältnis. Im Mittelpunkt der Betreuung steht der Mensch, aber auch die Familie und das soziale Umfeld werden mit einbezogen. Kommunikation, die sowohl den Informationsaustausch, das Empowerment, die Sensibilität für die Bedürfnisse der Betroffenen und der Partnerschaft als auch interprofessionelle Zusammenarbeit und Gesundheitsförderung (Fallmanagement, effizienter Einsatz von Ressourcen) umfasst, sind wichtige Merkmale.

\section{Finanzierung des Gesundheitssystems}

Die Finanzierung des «Medical Home» beruht auf zwei Systemen: "Mutualité» (auf Gegenseitigkeit beruhende Versicherung) und auf einer Patientenpauschale. Das Prinzip der "Mutualité» ist die Selbstverwaltung. Sie verfolgt im Interesse ihrer Mitglieder einen gemeinnützigen Zweck gegen Zahlung eines Beitrags sowie Leistungen der Prävention, der Solidarität und der gegenseitigen Hilfe. Die Beiträge werden von den im «Medical Home» behandelten Personen bezahlt, entsprechend ihrer finanziellen Leistungsfähigkeit, d.h. nach ihrem Einkommen.

Die Reform basiert auf einer integrierenden Sichtweise, neuen Formen der Finanzierung und einer unabhängigen Qualitätskontrolle.

Keine Tätigkeit des "Medical Home» wird pro ausgeführte Leistung vergütet, um unnötige, durch Gewinn motivierte Eingriffe zu vermeiden. Die Bezahlung erfolgt nach Kopfpauschale (Capitation), d.h. nach der Anzahl der behandelten Personen. Die Capitation umfasst nicht nur Hausärztinnen und -ärzte sowie das Pflegepersonal, sondern auch alle anderen beteiligten Akteure. So «kauft» die «Mutualité» Facharztkonsultationen, Krankenhaustage sowie Interventionen zu Hause, immer in Abhängigkeit von der Anzahl der im «Medical Home» Behandelten und entsprechend ihren Bedürfnissen. Dazu gehören auch bestimmte soziale Leistungen, da soziale Faktoren bei der Gesundheit oft eine wichtigere Rolle spielen als biologische.

Sobald der Beitrag bezahlt ist, ist der Zugang zu allen Leistungen kostenlos. Die Franchise, die derzeit mehr oder weniger hoch ist, wird vereinheitlicht und in die Prämien einbezogen. Behandlungen ausserhalb des «Medical Home» werden von der Krankenkasse nicht übernommen, ausser im Notfall oder mit ausdrücklicher Zustimmung der hausärztlichen Fachkraft. 


\section{Qualitätskontrolle}

Eine solche Organisation erfordert zwei Qualitätskontrollsysteme. Die von der "Mutualité» abgewickelten Finanzströme müssen von einer unabhängigen Institution kontrolliert werden, die jedoch dem Staat gegenüber verantwortlich ist (wie die FINMA in der Schweiz). In diesem Gremium sollten Patientenorganisationen vertreten sein.

Eine ähnliche Einrichtung muss die Qualität der Pflege kontrollieren. Die Herausforderung besteht darin, effizient und so unbürokratisch wie möglich zu sein. Am einfachsten wäre es, die Qualität der Versorgung vertikal, d.h. nach den Pathologien (z.B. Blutdruckwerte) zu kontrollieren. So wird es in England mit dem «Quality and Outcomes Framework» gemacht [5]. Schwieriger zu erreichen, aber relevanter ist die "horizontale» Qualitätskontrolle, d.h. die Messung der von Patientinnen und Patienten bewerteten Ergebnisse. Solche Überwachungssysteme sind wegen fragwürdiger Ergebnisse und der damit einhergehenden Bürokratie kritisiert und teilweise aufgegeben worden. Die Qualitätskontrolle innerhalb des «Medical Home» sollte auf der Verantwortung der Leistungserbringer durch Qualitätszirkel entsprechend ihrer Deontologie und Ethik beruhen und sich dabei auf den aktuellen Wissensstand stützen (evidenzbasierte Medizin). Ein Jahresbericht, der an das für die Qualitätskontrolle zuständige externe Gremium gerichtet wird, würde von dieser Verantwortung zeugen. Für die externe Kontrolle sollten innovative Methoden gefunden werden: Die Herausforderung wäre, mittels personalisierter Interviews die Verbesserung des Gesundheitszustands der Patientinnen und Patienten im Hinblick auf ihre Fähigkeit zum Selbstmanagement und zur Anpassung an ihre Lebensumstände zu messen.

\section{Beispiele für eine solche Organisation des Gesundheitswesens}

Auf internationaler Ebene nähern sich einige Gesundheitssysteme dem PCC. In England bietet der National Health Service (NHS) kostenlose Gesundheitsversorgung für alle. Im Zuge der Implementierung des NHS wurden Richtlinien und Qualitätskontrollsysteme eingeführt. Im Grossen und Ganzen wird der NHS von der Bevölkerung geschätzt. Das Problem ist, dass der Service als staatliches Gesundheitssystem von staatlichen Ressourcen abhängig ist, die je nach dem momentanen politischen Trend variieren können. Ein ähnliches System findet sich in Australien. In den Vereinigten Staaten haben Unternehmen wie Kaiser-Permanente eine Versorgung nach dem PCC-Prinzip zu angemessenen
Kosten ermöglicht. In der Schweiz schliesslich kommen Institutionen wie die Cité Générations - Maison de Santé in Onex (GE) oder das Center da sandà Engiadina bassa (GR) unseren Vorschlägen am nächsten [6]. Alle diese Organisationen haben zur Kostensenkung beigetragen. Keine von ihnen erfüllt jedoch alle hier gelisteten Anforderungen, d.h. eine auf Gegenseitigkeit beruhende Krankenversicherung, eine Kopfpauschale für alle Teilnehmenden oder die Unabhängigkeit vom Staat.

\section{Bedingungen für den Erfolg der Reform}

Unser Vorschlag basiert auf fünf Prinzipien: Gegenseitigkeit der Finanzierung mit Beiträgen entsprechend dem Einkommen, Vergütung aller Anbieter entsprechend der Kopfpauschale, freier Zugang zur Versorgung und ein System der internen Kontrolle auf der Grundlage der Verantwortung und der externen Kontrolle entsprechend den Ergebnissen der Gesundheitsverbesserung. Ausserdem sollen die Ziele mit so wenig Bürokratie wie möglich erreicht werden.

\section{Solidarische Finanzierung}

Gemäss dem Bundesgesetz betreffend die Aufsicht über die soziale Krankenversicherung (Krankenversicherungsaufsichtsgesetz, KVAG) müssen die Versicherer mehrere Bedingungen erfüllen (Art. 5), insbesondere müssen sie «die Rechtsform der Aktiengesellschaft, der Genossenschaft, des Vereins oder der Stiftung aufweisen» (Art. 5a) sowie «die soziale Krankenversicherung nach dem Grundsatz der Gegenseitigkeit durchführen und die Gleichbehandlung der Versicherten gewährleisten» (Art. 5f).

Zugelassene Krankenversicherungen [7] erfüllen zwar diese rechtlichen Forderungen, aber sie sind vor allem Aktiengesellschaften, deren Hauptzweck darin besteht, Gewinne zu erwirtschaften. Es besteht daher im KVAG ein gewisser Widerspruch zwischen Artikel 5a und 5f. Ein einkommensabhängiger Beitrag wurde von zwei Initiativen vorgeschlagen, aber vom Parlament und der Bevölkerung abgelehnt. Die Schweiz ist das einzige Land in Europa, das dieses System nicht kennt, was bedeutet, dass der Staat Personen mit niedrigem Einkommen bei der Zahlung der Beiträge unterstützen muss. Die Einführung eines solchen Systems wäre ideal und würde Verwaltungskosten sparen, aber es ist für die Verwirklichung unseres Projekts nicht unerlässlich.

\section{Pro-Kopf-Finanzierung}

Die Einführung der Patientenpauschale (Capitation) für alle Aktivitäten ist ein wesentlicher Bestandteil der Reform. Sie bezieht nicht nur auf die Hausärztinnen 
und -ärzte, wie dies bereits in einigen HMOs in der Schweiz praktiziert wird, sondern auch auf Spezialistinnen und Spezialisten, Krankenhausaufenthalte, Hauspflege und bestimmte soziale Aktionen. Patientenpauschalen reduzieren die Bürokratie, da detaillierte Rechnungen - Tarmed ambulant, DRG stationär - nicht mehr notwendig sind. Darüber hinaus werden durch finanziellen Gewinn motivierte Handlungen vermieden. Die Kopfpauschalenabrechnung ist unter Art. 43 c) des Krankenversicherungsgesetzes (KVG) für alle Leistungserbringer vorgesehen.

\section{Freier Zugang zur Versorgung}

In der Schweiz ist die Kostenbeteiligung die höchste in Europa. Sie besteht aus der Franchise von 300 bis 2500 CHF und dem zehnprozentigen Selbstbehalt für alle Leistungen. Infolgedessen verzichtet derzeit ein erheblicher Teil der Bevölkerung aus Kostengründen auf gesundheitliche Versorgung [8]. Es sind oft diejenigen, die sie am meisten benötigten [9]. Einige Parlamentsmitglieder möchten die Franchise noch weiter erhöhen. Dies steht im Gegensatz zu den jüngsten wissenschaftlichen Erkenntnissen [u.a. 10], die zeigen, dass der freie Zugang zur Gesundheitsversorgung zwar die Zahl der Konsultationen erhöht, aber die Spitalaufenthalte und damit die Gesamtkosten verringert.

\section{Qualitätskontrolle}

Qualitätskontrolle ist in jedem Gesundheitssystem unerlässlich. Sie sollte die Finanzströme und die Aktivitäten der Anbieter kontrollieren. Gegenwärtig ist das BAG für die Kontrolle der Krankenkassen zuständig. Wir sind der Meinung, dass der Staat nicht das Gesundheitssystem selbst kontrollieren, sondern die Kontrolle an eine Kommission delegieren sollte, nach dem Vorbild der FINMA: eine unabhängige Organisation, die aber dem Staat gegenüber verantwortlich ist. Die Qualitätskontrolle der Dienstleistenden sollte in gleicher Weise von einer unabhängigen Kommission organisiert werden. Ein Gesetz in dieser Richtung ist derzeit in Vorbereitung [11].

\section{Abbau von Bürokratie}

Die derzeitige Organisation des schweizerischen Gesundheitswesens erfordert einen so hohen administrativen Aufwand des medizinischen Personals, dass dieser die für Behandlungen aufgewendete Zeit zu übersteigen droht. Dies hat zur Folge, dass Ärzte überdurchschnittlich von Burn-out betroffen sind [12] und fast die Hälfte der Pflegefachpersonen ihren Beruf aufgeben [13]. Unser Reformprojekt ermöglicht es, die Bürokratie erheblich zu reduzieren, da die Rechnungsstellung und die Qualitätskontrolle mit einem Minimum an Verwaltung verbunden sind.

\section{Bildnachweis}

(c) Palto | Dreamstime.com

\section{Literatur}

1 Constand, M.K., MacDermid, J.C., Dal Bello-Haas, V. et al. Scoping review of patient-centered care approaches in healthcare. BMC Health Serv Res 14, 271 (2014). https://doi.org/10.1186/1472-6963-14-271

2 Santana MJ, Manalili K, Jolley RJ, Zelinsky S Quan H, Lu M. How to practice person centred care: A conceptual framework. Health Expect. 2018 Apr; 21(2): 429-40.

3 Rosenthal TC. The Medical Home: Growing evidence to support a new approach to primary care. J Am Board Fam Med 2008; 21:42740.

4 Price RA, Elliott MN, Cleary PD, Zaslavsky AM, Hays, RD. Should health care providers be accountable for patients' care experiences? Gen Intern Med 2014;30:253-6 DOI: 10.1007/s11606-014-3111-7

5 Roland M, Guthrie B. Quality and outcomes framework: what have we learnt? BMJ 2016;354:14060

6 Rippstein J. Nous devons sortir d'une médecine centrée sur l'hôpital. Bulletins des médecins suisses. 2020;101:384-6

7 bag.admin.ch/dam/bag/fr/dokumente/kuv-aufsicht/bakv/ Zugelassene-krankenversicherer/zugelassenen-krankenversicherer-0120.pdf.download.pdf/Zugelassene\%20Krankenversicherer_1_01_2020.pdf

8 Bundesamt für Statistik BFS. Gesundheitszustand, Armut und Verzicht auf Pflegeleistungen. Erhebung über die Einkommen und die Lebensbedingungen (SILC) 2011. Neuenburg 2013.

9 Wolff H, Gaspoz JM, Guessous I. Health care renunciation for economic reasons in Switzerland.SwissMedWkly.2011;141:w13165. DOI: https://doi.org/10.4414/smw.2011.13165

10 Moloo H, Mor V. Increased ambulatory care copayments and hospitalizations among the elderly. N Engl J Med 2010;362:320-8.

11 bag.admin.ch/bag/fr/home/versicherungen/krankenversicherung/kranken-versicherung-revisionsprojekte/netzwerkqualitaet-gesundheitsversorgung.html

12 Kursner D, Danuser B Arbeitsmedizin: Die Arbeitsmedizin nimmt die psychische Gesundheit der Schweizer Ärztinnen und Ärzte unter die Lupe. Schweiz Med Forum 2007;7:7-8

13 https://www.obsan.admin.ch/de/publikationen/berufsaustrittevon-gesundheitspersonal.

\section{Das Wichtigste in Kürze}

- Die Patientinnen und Patienten stehen im Mittelpunkt der Reform. Das «Medical Home» ermöglicht es, alle Aspekte der Pflege zu organisieren.

- Die Finanzierung der Reform basiert auf Gegenseitigkeit. Alle Leistungen, einschliesslich Facharztkonsilien, Spitalaufenthalte und Hauspflege, werden durch Kopfpauschalen bezahlt. Die Vorschläge haben eine Kostensenkung zur Folge.

- Die Kontrolle wird durch nichtstaatliche, aber dem Staat gegenüber verantwortliche Aufsichtskommissionen organisiert. 